\title{
DriverPower: Combined burden and functional impact tests for cancer driver discovery
}

\author{
Shimin Shuai ${ }^{1,4}$, Steven Gallinger ${ }^{2,3}$ \& Lincoln Stein ${ }^{1,4}$ on behalf of the PCAWG Drivers and Functional \\ Interpretation Group and the ICGC/TCGA Pan-Cancer Analysis of Whole Genomes Network \\ ${ }^{1}$ Department of Molecular Genetics, University of Toronto, Toronto, Ontario, Canada \\ ${ }^{2}$ Lunenfeld-Tanenbaum Research Institute, Mount Sinai Hospital, Toronto, Ontario, Canada \\ ${ }^{3}$ Division of General Surgery, Toronto General Hospital, Toronto, Ontario, Canada \\ ${ }^{4}$ Informatics Program, Ontario Institute for Cancer Research, Toronto, Ontario, Canada
}

\section{Abstract}

2 We describe DriverPower, a software package that uses mutational burden and functional impact evidence to 3 identify cancer driver mutations in coding and non-coding sites within cancer whole genomes. Using a total of

4 1,373 genomic features derived from public sources, DriverPower's background mutation model explains up to $593 \%$ of the regional variance in the mutation rate across a variety of tumour types. By incorporating functional 6 impact scores, we are able to further increase the accuracy of driver discovery. Testing across a collection of 2,583 7 cancer genomes from the Pan-Cancer Analysis of Whole Genomes (PCAWG) project, DriverPower identifies 217 8 coding and 95 non-coding driver candidates. Comparing to six published methods used by the PCAWG Drivers 9 and Functional Interpretation Group, DriverPower has the highest F1-score for both coding and non-coding driver 10 discovery. This demonstrates that DriverPower is an effective framework for computational driver discovery.

\section{Introduction}

12 Cancer drivers are somatic genetic alterations that confer selective advantages to tumour cells $\mathrm{s}^{1,2}$. 13 Identification of cancer drivers is a crucial yet challenging task in cancer genomics research ${ }^{3,4}$. 14 There are multiple challenges. First, driver mutations generally account for only a small fraction 15 of the somatic variations found in a typical tumour, the rest being innocent bystander "passenger" 16 mutations $^{5}$. Second, there is substantial intra- and inter-tumoural heterogeneity in most cancers ${ }^{6}$. 17 Both across different tumour types and across different genomic regions within the same tumour, 18 the background mutation rate can vary over several orders of magnitude.

19 The advent of large scale cancer whole-genome sequencing (WGS) data, such as the 2,600 20 tumour and matched normal whole genomes from the ICGC/TCGA Pan-Cancer Analysis of Whole 21 Genomes (PCAWG) project, has made it possible to explore the role of driver events in non22 coding regions. However, identifying non-coding driver events in WGS creates new challenges. 
23 First, while the functional impact of somatic mutations in the coding regions of genes is fairly

24 straightforward to predict, much less is known about the effect of mutations on non-coding

25 regions of the genome. Second, only $\sim 1 \%$ of somatic mutations detected in PCAWG WGS data

26 are exonic, adding substantially more mutations and regions to be tested and demanding more

27 careful control of type I and type II errors than whole exome sequencing. At present, only a limited

28 number of non-coding drivers are known, the primary examples being the TERT promoter for

29 multiple tumour types and the TAL1 enhancer for $T$ cell acute lymphoblastic leukaemia ${ }^{7,8}$.

30 Most state-of-the-art methods identify drivers by detecting signals of positive selection either

31 through mutational burden tests, which compare the rate of mutations observed in a region of

32 the genome to what is expected from the background mutation rate, or functional impact tests,

33 which identify putative driver mutations based on a higher-than-expected rate of changes that

34 are predicted to alter the function of genomic elements ${ }^{3,6}$. Mutational burden tests work best for

35 calling frequently recurrent driver events and perform poorly when applied to rare driver events.

36 In contrast, functional impact tests fail to find drivers in genomic elements that are poorly

37 understood or annotated. To maximize accuracy, we combined the two mutation significance

38 testing methods to develop DriverPower (Fig. 1a), a framework for identification of coding and

\section{Results}

41 To evaluate DriverPower, we took WGS somatic variant data derived from 2,583 high-quality 42 donors from the PCAWG project ${ }^{9}$. After removing hypermutated samples, 2,514 donors with $4324,715,214$ somatic single nucleotide variants (SNV) and small indels were used for driver element 44 identification. We analysed this data both as a single pan-cancer data set, as well as a series of 4529 tumour type-specific cohorts (Supplementary Table 1).

\section{Features predictive of background mutation rate}

47 Among all tumour cohorts, we observed substantial variability in the observed mutation rate at the tissue, donor and locus levels (Supplementary Fig. 1 and 2). Accurate driver detection requires an accurate estimate of background mutation rate across the tumour genome, taking into account the extensive variability among tumour types, donors and genomic regions. DriverPower tackles this issue by modelling the background mutation rate (BMR) using numerous genomic features that co-vary with the localized background mutation rate. We collected 1,373 features from three public data portals (Supplementary Table 2): the ROADMAP Epigenomics project, the ENCODE project and the UCSC genome browser ${ }^{10,11,12}$. These features covered seven main categories: conservation, DNA accessibility, epigenomic marks, nucleotide contents, replication timing, RNA expression and genome compartments. As expected, we found extensive multicollinearity among features. Most features (1368/1373) are significantly correlated with pan-cancer genome-wide mutation rates (Spearman's rho test $q<0.1$; Supplementary Fig. 3). 


\section{Background mutation rate model}

60 We investigated two algorithms for modelling the BMR based on genomic features. The first algo-

61 rithm was randomized lasso followed by binomial generalized linear model (GLM). The alternative

62 algorithm was the gradient boosting machine (GBM), which is a non-linear and non-parametric

63 tree ensemble algorithm ${ }^{13}$. To evaluate both BMR modelling algorithms, we made non-overlapped

641 megabase pair (Mbp) autosomal elements $(n=2,521)$ as well as training genomic elements

$65(n=867,266)$ by sampling genomic coordinates randomly. The number of mutations per element

66 was then predicted with 5-fold cross-validation.

67 When evaluated using $1 \mathrm{Mbp}$ autosomal elements, we found that both algorithms could accurately

68 predict the background mutation rate (Supplementary Fig. 4 and 5). In high mutational

69 burden tumour cohorts, we observed essentially no difference between two algorithms, however

70 GBM consistently outperformed GLM when applied to low mutational burden tumour cohorts

71 (Supplementary Fig. 6). When evaluated on the training element set, in which the size of

72 element varies from $100 \mathrm{bp}$ to $1 \mathrm{Mbp}$, the prediction accuracy drops due to higher BMR variability,

73 especially for low mutational burden tumour cohorts such as Myeloid-MPN and CNS-PiloAstro

74 (Supplementary Fig. 6). However, for large cohort such as the pan-cancer set $(\mathrm{N}=2,253)$,

75 around $93 \%$ of the mutation rate variance on the training set is explained by either model (Fig.

76 1b). The model still shows excellent performance when applied to the test element set, explaining

$7783 \%$ of the mutation rate variance on the pan-cancer cohort (Fig. 1c).

78 Both the randomized lasso algorithm and the GBM can be used to rank feature importance in 79 different ways. Feature selection ranking from both methods confirmed that H3K9me3 (associ-

80 ated with heterochromatin), replication timing and H3K27ac (or its antagonistic histone mark 81 H3K27me3) are the most important groups of predictors for BMR (Supplementary Fig. 7

82 and Supplementary Table 2$)^{14}$. Consistent with previous results, we found that features from 83 tumour cell lines with similar cell-of-origin to the primary tumour type are frequently selected ${ }^{15}$.

84 For example, replication timing from liver cancer cell line HepG2 was selected as a feature for the 85 BMR in hepatocellular carcinoma (Liver-HCC), while replication timing in MCF7 (breast cancer)

86 and SK-N-SH (neuroblastoma) were selected for breast adenocarcinoma (Breast-AdenoCA) and 87 glioblastoma (CNS-GBM), respectively (Supplementary Fig. 8).

\section{Functional adjustment}

89 In most burden-based methods mutations are equally weighted. However, not all mutations 90 have the same functional consequences. To incorporate functional consequence information, 91 DriverPower implements a posterior functional adjustment. The functional adjustment step up92 weights mutations with high predicted functional impact. While the DriverPower framework can 93 potentially work with any functional scoring scheme, in the current implementation we measured 94 the functional impact using four published scoring schemes: the CADD ${ }^{16}$, DANN $^{17}$, EIGEN $^{18}$ and 95 LINSIGHT $^{19}$ scores. Although different training data, assumptions and algorithms are used by 96 different scores, we found those scores to be consistent at the element level (Supplementary 97 Fig. 9). We used the average weight of all four scores in the remainder of the manuscript unless 
otherwise specified.

\section{Candidate driver event discovery}

To evaluate the DriverPower algorithm, we first employed three simulated variant sets generated by the PCAWG Drivers and Functional Interpretation Group (PDFIG) to examine type I and type II errors. We expected to identify no drivers as all three simulated datasets are reshuffles of observed mutations. In general, we observed no inflation or deflation in simulations and only 8 significant hits were identified in $\sim 11 \mathrm{M}$ statistical tests (Supplementary Fig. 10). We then used the observed PCAWG dataset to discover drivers within multiple coding and non-coding element sets identified by the PDFIG, spanning 3.7\% ( ${ }^{113} \mathrm{Mbp}$ ) of the human genome.

We benchmarked our results against reference driver element sets and driver candidates called by six other published methods. Among the six methods, ExInAtor ${ }^{20}$, ncdDetect ${ }^{21}$ and LARVA ${ }^{22}$ use only mutational burden information; oncodriveFML ${ }^{23}$ uses only functional biases; while MutSig ${ }^{24}$ and ActiveDriverWGS ${ }^{25}$ model both mutational burden and functional consequence but not through functional impact scores. Three reference driver element sets were used: the COSMIC Cancer Gene Census (CGC) ${ }^{26,27}$, the PCAWG raw integrated driver candidates (PCAWG-raw) and the PCAWG consensus driver candidates (PCAWG-consensus). The CGC is a catalogue of driver genes for which mutations have been causally implicated in cancer and was used as the gold standard set (i.e., used in the calculation of precision and recall) for coding and splice site drivers. PCAWG-raw is an integration of driver elements called by 12 different driver detection methods on the same data we used here. PCAWG-consensus is a conservative set derived from the PCAWG-raw by applying multiple stringent filters to control the false discovery rate; in particular, the majority of non-coding candidates from lymphoid tumours and skin melanomas is excluded from this set because of hyper-mutational processes in these tumour types that create prominent mutational hotspots ${ }^{28,29,30}$. For the same reason our analysis of non-coding regions for tumour-specific and the pan-cancer cohorts excluded melanoma and lymphoma.

Overall, we observed well calibrated p-values in DriverPower's results with or without functional adjustment (Fig. 1d and Supplementary Fig. 10) and a high accuracy for both coding and non-coding driver discovery (Fig. 1e, Supplementary Table 3). For protein coding regions (CDS), DriverPower found 217 significant driver candidates. Since a gene (e.g., TP53) can be driver in multiple cohorts, the unique number of genes was 131 . The precision of the algorithm's driver calls was high. Among the driver genes called by DriverPower, $82.5 \%$ (179/217) of all genes were present within the CGC. For non-CGC genes, 27 and 3 genes were present within PCAWGconsensus and PCAWG-raw, respectively. Thus, only $3.7 \%$ (8/217) coding driver candidates called by DriverPower were not contained within any reference gene sets. As expected, incorporation of functional information increased both precision and recall in coding driver discovery (Fig. 2a and Supplementary Fig. 11). For example, in pancreatic ductal adenocarcinoma (PancAdenoCA; $\mathrm{N}=232$ ), the addition of functional adjustment to the algorithm resulted in a gain of three additional drivers (ACVR1B, RBM10 and ZFP36L2) and the loss of one likely false positive genes $(F A U)$ (Fig. 2a). Without the use of functional information, the overall precision 
137 dropped to $74.6 \%(156 / 209)$ for CGC genes and $88.5 \%(185 / 209)$ for CGC/PCAWG genes.

138 When compared to six other methods using the same 26 non-melanoma/lymphoma cohorts and

139 CGC as the gold standard set, DriverPower (precision=0.84; recall=0.79) had the highest F1-

140 score (0.81) (Fig. 2b, c). In our benchmark, sensitivity was a bottleneck for most methods (4/7

141 with recall $<0.5)$. When compared to the method with highest recall, the widely used coding

142 driver caller MutSig (precision=0.80; recall=0.80), DriverPower identified an additional 21 genes

143 present in CGC (23 for MutSig; Supplementary Fig. 12).

144 We next benchmarked DriverPower's accuracy for non-coding driver events. For the prediction 145 of driver events affecting the splice sites of coding genes, DriverPower called 47 significant can146 didates with 85.1\% (40/47) within CGC. DriverPower ( $F 1=0.91)$ also outperformed two recently 147 published methods, ncdDetect $(F 1=0.65)$ and oncoDriverFML $(F 1=0.32)$, for splice site driver 148 detection (Supplementary Fig. 13).

149 For the prediction of non-coding driver events in 3'UTRs, 5'UTRs, promoters and enhancers, 150 DriverPower identified 19 candidates in non-melanoma/lymphoma tumour cohorts and 24 can151 didates in the pan-cancer cohort. Benchmarking results showed that DriverPower has the high152 est F1-score (0.79) among the six methods evaluated (Fig. 2d, e). Promoter and 5'UTR 153 driver candidates called by DriverPower were associated with a total of 17 unique genes. Of 154 these, one gene (TERT) was in CGC, four genes (WDR74, HES1, MTG2 and PTDSS1) were in 155 PCAWG-consensus, and six other genes were in PCAWG-raw. DriverPower also called two 3'UTR 156 driver candidates in total, including TOB1 in pan-cancer and $A L B$ in Liver-HCC. Both candi157 dates were present in the PCAWG-consensus. For enhancer regions, DriverPower identified two 158 candidates: chr6:142,705,600-142,706,400 (linked to GPR126), and chr7:86,865,600-86,866,400 159 (linked to TP53TG1). Both enhancer elements were identified by PCAWG-raw; the TP53TG1 160 enhancer was the only enhancer for non-melanoma/lymphoma tumours in the PCAWG-consensus 161 set.

162 For long non-coding RNA (IncRNA) genes and their promoters, DriverPower found 9 candidates 163 in total. Among them, 6 and 3 were contained within PCAWG-consensus and PCAWG-raw, 164 respectively. These candidates targeted three unique IncRNAs: RN7SK, RMRP and RPPH1. 165 The promoter of RMRP was significantly mutated in four cohorts (Breast-AdenoCA, Liver-HCC, 166 Stomach-AdenoCA and pan-cancer) and has been nominated as a novel non-coding driver.

\section{DriverPower-exclusive driver candidates overview}

168 A total of 11 coding and 17 unique non-coding candidates were exclusively identified by Driver169 Power (not present in either CGC or PCAWG-consensus; Supplementary Table 4). We sought 170 to evaluate these exclusive driver candidates using literature evidence and correlative orthogonal 171 data such as the effect of the variant on RNA-seq expression levels and the presence of somatic 172 copy number alterations (SCNAs) and somatic structural variations (SVs) covering the same re173 gions. On this basis, we found that many of the DriverPower-exclusive candidates are plausible 174 cancer drivers. 
175 Among protein coding genes, DriverPower identified EEF1A2 (eukaryotic translation elongation 176 factor 1 alpha 2) in the esophageal adenocarcinoma cohort (Eso-AdenoCA; 7/95 samples). All 177 seven observed mutations were missense (Supplementary Fig. 14a). Although no RNA-seq 178 data is available for Eso-AdenoCA samples, SCNA analysis indicated that EEF1A2 is amplified in $17969.5 \%$ (66/95) of Eso-AdenoCA samples (vs. 27.9\% of non-Eso-AdenoCA samples; Supplemen180 tary Fig. 14b), suggesting a potential gain-of-function role in this cancer type. The amplification 181 of EEF1A2 (20q13.33) was also confirmed by the GISTIC2.0 ( $q=0.0006)$. The same $1 \mathrm{Mbp}$ locus 182 detected by GISTIC2.0 was also amplified recurrently in other tumour types, including $73.1 \%$ of 183 colorectal adenocarcinoma, $64.7 \%$ of stomach adenocarcinoma and $55.4 \%$ of ovarian adenocar184 cinoma. Supporting this hypothesis, previous studies have also demonstrated that EEF1A2 is a 185 putative oncogene in ovarian cancer and overexpressed in various tumour types ${ }^{31,32,33}$.

186 Another protein coding gene exclusively identified by DriverPower was $M E F 2 B$ in B-cell non187 hodgkin's leukaemia (Lymph-BNHL; 8/105 samples). Among 9 observed mutations, 8 mutations 188 were missense and one was a frameshift deletion (Supplementary Fig. 14c). RNA-seq data 189 confirmed that mutated samples overexpressed MEF2B (copy number corrected $p=0.011$; Sup190 plementary Fig. 14d). MEF2B (Myocyte enhancer factor 2B) has been identified in multiple 191 WES studies ${ }^{34,35,36}$, and a previous study has also shown that MEF2B mutations can dysregulate 192 cell migration in non-Hodgkin lymphoma ${ }^{37}$.

193 One splice site candidate exclusively called by DriverPower is SGK1 (serum/glucocorticoid regu194 lated kinase 1) in Lymph-BNHL. The same gene was also significant in DriverPower's CDS result 195 for Lymph-BNHL (Supplementary Fig. 14e), resulting in a total of $13.3 \%(14 / 105)$ Lymph196 BNHL samples being affected by non-synonymous or splice site mutations in SGK1. SGK1 is 197 present in PCAWG-raw but was filtered out due to the large number of AID-related variants in 198 this tumour cohort. However, differential expression analysis indicated that SGK1 is significantly 199 overexpressed in mutated Lymph-BNHL samples relative to non-mutated samples (copy number 200 corrected $\mathrm{p}=3 \mathrm{e}-13$; Fig. 2f). SGK1 encodes a serine/threonine protein kinase that plays an im201 portant role in cellular stress response and its CDS has been nominated as a driver in earlier WES 202 studies $^{35,36}$. Another study has also demonstrated that the administration of an SGK1 inhibitor 203 induces apoptosis in lymphoma cell lines ${ }^{38}$. Together these data support a potential driver role 204 for SGK1 in Lymph-BNHL.

205 The GPR126 (adhesion G protein-coupled receptor G6) enhancer candidate was filtered out 206 from the PCAWG-raw set because of mutations in palindrome loops, which makes it unclear 207 whether mutations in the GPR126 enhancer are caused by mutational mechanism associated 208 with palindrome loops or positive selection. We found that the GPR126 enhancer is recurrently 209 mutated in transitional cell carcinoma of the bladder (Bladder-TCC; 14/23 samples) and breast 210 adenocarcinoma (Breast-AdenoCA; 8/195) (Supplementary Fig. 14f). GPR126 is among 211 the MammaPrint $R 70$ gene panel used to predict the risk of breast cancer metastasis ${ }^{39,40}$. A 212 study also shows that knockdown of GPR126 can inhibit the hypoxia-induced angiogenesis in 213 model organisms ${ }^{41}$. Differential expression analysis demonstrated that the GPR126 is signifi214 cantly downregulated in Bladder-TCC samples with enhancer mutations (copy number corrected $215 \mathrm{p}=0.012$; Fig. $\mathbf{2} \mathbf{g}$ ) relative to those carrying the wild type enhancer, suggesting a functional role 
216 for these mutations.

217 Several somatically altered histone genes have been implicated in human cancer, such as $H 3 F 3 A$ 218 (identified as a pan-cancer driver in this study), $H 3 F 3 B$ and $H I S T 1 H 3 B^{42,43,44}$. DriverPower iden-

219 tified four histone genes as driver candidates in the pan-cancer cohort, two of which were absent 220 from CGC or PCAWG-consensus: the 5'UTR of HIST1H2AC and HIST1H2BD. Previous studies 221 have shown that the protein levels of the replication-dependent histone $\mathrm{H} 2 \mathrm{~A}$ variant HIST1H2AC 222 (encoding histone $\mathrm{H} 2 \mathrm{~A}$ type $1-\mathrm{C}$ ) is decreased in chronic lymphocytic leukaemia patients and 223 bladder cancer cells ${ }^{45,46}$, and the siRNA knockdown of HIST1H2AC increases cell proliferation 224 and promote oncogenesis ${ }^{46}$.

225 Several other driver candidates exclusively called by DriverPower are associated with genes that 226 may have a role in cancer. The highly expressed liver-specific gene $A L B$ (albumin) is significant 227 for somatic mutations affecting its CDS, splice site, 3'UTR and promoter in Liver-HCC; the 228 splice site and promoter (under CADD scores) were discovered by DriverPower exclusively. Cor229 relative evidence from DNA promoter methylation, gene expression and copy number alterations 230 suggested that loss-of-function mutations in $A L B$ are subject to positive selection in Liver-HCC 231 as described in ref. xx (Driver group main paper). The CDS of KAT8 (lysine acetyltransferase 232 8) was called by DriverPower in Panc-AdenoCA with $100 \%$ (5/5) missense mutations. As a 233 histone modifier, KAT8 has been shown to physically interact with $M L L 1$ and regulate known 234 cancer drivers ATM and TP5347,48,49,50. Previous studies have also shown that KAT8 is down235 regulated in gastric cancer ${ }^{51}$ and $K A T 8$ can suppress tumour progression by inhibiting epithelial236 to-mesenchymal transition ${ }^{52}$. The 5'UTR and promoter of SRSF9 (serine and arginine rich splicing 237 factor 9) was significant in DriverPower's results for pan-cancer and not present in any reference 238 driver sets. The protein encoded by SRSF9 is part of the spliceosome; a previous study indicates 239 that the proto-oncogene SRSF9 is overexpressed in multiple tumours and that this overexpressi240 on can cause the accumulation of $\beta$-Catenin ${ }^{53}$. The same study also showed that the depletion 241 of SRSF9 proteins could inhibit colon cancer cell proliferation.

242 In summary, 4/11 coding and 4/17 unique non-coding driver candidates exclusively called by 243 DriverPower had some form of support from the literature or orthogonal evidence. If we assume 244 that all the exclusive candidates that lack such evidence are false positives, then this puts an 245 estimate of DriverPower's false discovery rate across the PCAWG data set at 3.2\% (7/217) 246 for coding and $16.8 \%$ (16/95) for non-coding regions. However, this assumption is probably 247 invalid as most of these lack-of-evidence candidates are also identified by other methods and 248 present in PCAWG-raw. We acknowledge that lack-of-evidence candidates may contain false 249 positive calls, but they may also contain previously unknown drivers. For example, the 5'UTR 250 of TBC1D12 in Breast-AdenoCA, which has been filtered out from the PCAWG-raw due to 251 possible hypermutability, is called by all but one driver discovery methods and is reported as 252 a putative cancer driver in previous studies because of two recurrent mutations in the Kozak 253 consensus sequence involving in the initiation of protein translation ${ }^{23,54}$. Moreover, according to 254 another recent study, the same TBC1D12 candidate is still statistically significant in breast cancer 255 even after removing hypermutations, but whether these mutations can alter protein translation 256 in cancer is still undetermined ${ }^{24}$. Some lack-of-evidence candidates may also fit the mini-driver 
257 model of cancer evolution ${ }^{55}$. Unlike classical drivers, mini-drivers can only weakly promote and 258 are not essential for tumour progression, hence present at a lower frequency in cancer cohorts.

259 Further investigation is required to determine the role of lack-of-evidence candidates in cancer.

\section{DriverPower applied to whole exome sequencing}

261 To demonstrate the robustness of DriverPower, we applied DriverPower to two public whole262 exome sequencing (WES) datasets (Supplementary Fig. 15). Both WES datasets are processed 263 differently than the PCAWG data and contain samples not included in the PCAWG study. For liver 264 cancer, using models trained for Liver-HCC $(\mathrm{N}=314)$, DriverPower identified 14 coding drivers 265 from 364 TCGA-LIHC samples ( 53 shared with Liver-HCC). All but one driver candidates were 266 present within the CGC or PCAWG-consensus. For pancreatic adenocarcinoma, using models 267 trained for Panc-AdenoCA $(\mathrm{N}=232)$, DriverPower identified 6 coding drivers from 180 TCGA268 PAAD samples (no shared samples with the PCAWG study) and all corresponded to known driver 269 genes.

\section{Discussion}

271 Computational driver discovery is essential to distinguish driver from passenger mutations in 272 the coding and non-coding regions of whole cancer genomes. Here we report DriverPower, a 273 new framework for accurately identifying both types of driver mutation by combining mutational 274 burden and functional impact information. The method takes advantage of the large somatic 275 mutation sets produced by WGS technology to build an accurate global BMR model from more 276 than a thousand genomic features. This contrasts with methods that build a local BMR model 277 using selected or flanking regions. One advantage of this is that the method is not biased towards 278 coding regions, but uses the same model for coding and non-coding cancer driver discovery. 279 Another advantage is the method's high degree of modularity. DriverPower can potentially work 280 with any types of genomic element (contiguous or disjoint, coding or non-coding, proximate or 281 distal to genes), any regression algorithms for modelling BMR and any functional impact score 282 scheme. Although DriverPower is designed for WGS projects, it performs robustly in whole-exome 283 sequencing strategies as well.

284 In comparison to the other driver discovery methods evaluated by the PCAWG Drivers and Func285 tional Interpretation Group, DriverPower provides the best balance of precision and recall, al286 though is not always the top ranked method when either metric is considered independently (Fig. 287 2b,d). As discussed in Supplementary Note 1, DriverPower is parameterized to allow for ad288 justment of the precision-recall trade-off; in this study, we selected conservative parameters that 289 prioritize precision over recall especially for non-coding regions.

290 There are several ways in which the accuracy of DriverPower could be improved. One approach to 291 improve recall is to take into the account the potential presence of negative (purifying) selection 292 in the functional regions selected for testing. When the BMR model is trained, we use random 
293 genomic elements that are predominantly under neutral selection. However, the functional ele294 ments selected for testing are more likely to be under positive and/or negative selection ${ }^{56}$. The 295 observed mutation rate reflects the balance between positive and negative selection, and negative 296 selection at one site in the element will diminish the signal of positive selection at other sites, 297 reducing the sensitivity of the method as a whole. To our knowledge, no driver discovery tool 298 currently models the effect of negatively selected sites; future work aims to take this mechanism 299 into account.

300 The precision of the method can also be improved. False positive driver calls may be caused by 301 technical errors such as variant-calling artefacts that artificially increase the local mutation rate, 302 or by biological processes that are not captured by the BMR model such as regional differences 303 in activation-induced cytidine deaminase (AID) activity. These can potentially be mitigated by 304 incorporating into the BMR model additional features relevant to the technical and biological 305 processes. For example, incorporating read-level coverage, mapping and bias scores into the 306 BMR could help correct for regions prone to variant-calling artefacts, while features like the 307 number of palindrome loops and the fraction of mutations caused by AID per element could be 308 used to adjust for locally-acting hypermutation processes.

309 When applied to the PCAWG data set, DriverPower called nearly twice as many non-coding 310 driver events than coding ones, a ratio also observed by the PCAWG driver study. While this 311 unbalanced ratio may reflect cancer biology, there is also the possibility that it reflects, at least 312 in part, the technical challenge of sequencing and interpreting non-coding regions. Potential 313 artefacts include systematic undercalling of somatic variants in non-coding regions ${ }^{24}$, a problem 314 that could be rectified by deeper coverage. Another technical issue is raised by the fact that several 315 non-coding candidates are only significant in the pan-cancer cohort, suggesting that the data set 316 is statistically underpowered. To overcome this issue, we could either sequence more genomes 317 or reduce the size of the set of test elements by narrowing it to functional motifs or conserved 318 bases $^{57}$. Lastly, functional impact score schemes are currently biased toward coding mutations; 319 therefore, improved functional scoring schemes will also help us identify more functionally relevant 320 non-coding cancer drivers in the future.

321 A comprehensive catalogue of coding and non-coding cancer drivers will accelerate the clinical 322 translation of cancer genomic study to precision medicine. As more cancer genomes and more 323 cancer types are sequenced, a general and accurate framework for computational driver discovery 324 like DriverPower will become increasingly useful.

\section{Acknowledgements}

326 This work was supported in part by the Government of Ontario. 


\section{Methods}

328 Generation of cancer whole-genome somatic mutations. All DNA somatic single nucleotide 329 variations (SNVs) and indels for 2,583 donors were obtained from the PCAWG project (somatic 330 variant callset released October 2016$)^{9}$. For our analysis, donors with hypermutated signatures 331 were excluded $(n=69$, defined as more than 30 mutations per $M b)$. Otherwise, we used the 332 same type-specific $(\mathrm{n}=29)$ and pan-cancer (all tumour samples except Skin-Melanoma, Lymph333 NOS, Lymph-CLL and Lymph-BNHL) sample cohorts as the PCAWG Drivers and Functional 334 Interpretation Group (PDFIG; Supplementary Table 1).

335 Generation of simulated somatic mutations. We used three simulated datasets (Broad, 336 DKFZ and Sanger simulations) from the PDFIG (described in detail at ref. $x x$ ). These simulations 337 were made to capture the variation of background mutation rate and remove the signal of positive 338 selection through permutations of observed somatic mutations.

339 Generation of test and training genomic elements. We define a genomic element as the 340 collection of genome coordinates that defines one specific functional region of interest. For 341 example, the CDS element of TP53 is the combination of all protein coding regions in TP53.

342 We used eight test element sets in our analysis, including the CDS ( $n=20,185)$, splice site $343(n=18,729), 5^{\prime} U T R(n=19,369), 3^{\prime} U T R(n=19,188)$, promoter $(n=20,164)$, enhancer $(n=30,816)$, $344 \operatorname{IncRNA}(\mathrm{n}=5,580)$ and IncRNA promoter $(\mathrm{n}=5,373)$. All test element sets were obtained from 345 the PCAWG project. GENCODE v19 was used as the reference gene model when building those 346 sets $^{58}$. Non-coding RNA annotations were collected from multiple sources as described.

347 We constructed genomic element training sets by randomly sampling genome coordinates from hg19, the build used for PCAWG. The length of each training element was sampled from the length distribution of test elements and multiplied by a factor of 3 . Training elements overlapping test elements were removed. In total, 867,266 training elements were created and $\sim 54 \%$ $(1,545,491,997 \mathrm{bp})$ of the genome was covered.

Collection and generation of features. We collected 1,373 features in total. Details including data sources can be found at Supplementary Table 2. Nucleotide content features were calculated as the fraction of 2-mers and 3-mers in each genomic element. The number of 2mers and 3-mers was counted directly from genome sequences (hg19). For raw features in bigwig format (typically genome wide signals), we calculated the average signal strength of covered bases in each element using the bigWigAverageOverBed (v2) utility from the UCSC genome browser ${ }^{59}$. For raw features in BED format (typically narrow peaks of ChIP-seq data), we calculated the percentage of bases intersecting BED for each element with the BEDTools $(v 2.24 .0)^{60}$. All missing values in features were filled with 0.

361 The DriverPower outline. The main steps of the DriverPower (v1.0.0) framework are sum362 marized below. Details of each step are described in following sections. The difference between 363 v1.0.0 and the version used in the PDFIG data analysis freeze (April 2017) is discussed in Sup364 plementary Note 2. 
1. Scale features and/or filter out excluded regions.

2. Build the background mutation rate model using the gradient boosting machine, or randomized lasso followed by binomial generalized linear models. The purpose of the BMR model is to estimate the expected number of mutations $(\hat{y})$ for any genomic element. Namely, we want to obtain $\hat{y}_{i}=E\left(y_{i} \mid X_{i}, L_{i}\right)$ where $X_{i}$ and $L_{i}$ are the feature vector and length for the element $i$.

3. Conduct burden test with observed $(y)$ and predicted $(\hat{y})$ mutation counts, and perform multiple testing correction.

4. Adjust observed mutation counts $(y)$ based on functional impact scores for nearly significant elements $(q<0.25)$.

5. Re-assess the significance for nearly significant elements with functional adjusted mutation counts followed by multiple testing correction.

Scaling of features. Features were scaled with RobustScaler from scikit-learn (version 0.18$)^{61}$. Feature scaling was only conducted for randomized lasso and GLMs.

Definition of excluded regions. In this study, all bases in the excluded regions were removed before any analysis. The excluded region consists of three sets: (1) all $\mathrm{N}$ bases and gaps in the hg19 genome (fetched from the UCSC table browser ${ }^{12}$ ); (2) ENCODE consensus excludable regions (the DAC Blacklisted Regions track and the Duke Excluded Regions track from the UCSC genome browser $)^{62}$; (3) PCAWG low mappability regions (data retrieved from the PCAWG variant group). PCAWG low mappability regions are defined as regions callable in fewer than 556/1111 ( $50 \%)$ tumour-normal pairs. For each tumour-normal pair, a base is callable if there are more than $14 / 8$ high quality reads in tumour/normal WGS. In total, $2,806,377,226 \mathrm{bp}$, or $96.41 \%$ of the genome are defined as callable.

Feature selection with randomized lasso. To select features, we randomly sub-sampled $10 \%$ of the training set 500 times. Then for the $k$-th subset with size $N_{k}$, the following model was fitted $^{63}$ :

$$
\hat{\mathbf{w}}_{k}=\arg \min _{\mathbf{w}}\left(\frac{1}{N_{k}}\left\|\operatorname{logit}\left(\frac{y+1 / 2}{N \cdot L}\right)-\mathbf{X} \mathbf{w}\right\|_{2}^{2}+\alpha \sum_{i=1}^{1373} \frac{\left|w_{i}\right|}{b_{i}}\right)
$$

where $N$ is the total number of donors in the dataset, $\mathbf{X}$ is the feature matrix, $\mathbf{w}$ is the weight vector, $\alpha$ is the regularization parameter, and $b_{i}$ is the scaling factor. The regularization parameter $\alpha$ was determined by a 5 -fold cross-validated lasso with $33 \%$ of the training data. For the $k$-th sub-sampling, the $i$-th feature was selected if $\hat{w}_{k i} \geq 0.001$. The final feature importance score was calculated as the fraction of times that a feature was selected. Only features with score $>$ 0.5 were used in the GLM BMR model.

Prediction of the BMR with GLM. When using the generalized linear model, we modelled 
the observed mutations in each genomic element with a binomial distribution, that is

$$
y \sim \mathbf{B}(n, p) \text { with } n=N \cdot L \text { and } p=\hat{y} /(N \cdot L)
$$

399 where $y$ is the observed mutation count and $\hat{y}$ is the estimated mutation count. We used the 400 binomial generalized linear model to obtain $\hat{y}$ with the logit link function, that is

$$
\frac{\hat{y}}{N \cdot L}=\mathbf{E}\left(\frac{y}{N \cdot L} \mid \mathbf{X}^{\text {select }}\right)=\operatorname{logit}^{-1}\left(\mathbf{X}^{\text {select }} \boldsymbol{\beta}\right)
$$

401 where $\mathbf{X}^{\text {select }}$ is the selected feature matrix and $\beta$ is the regression coefficient vector.

402 Prediction of the BMR with GBM. We trained the gradient boosting machine with XGBoost ${ }^{64}$. 403 All features were used in model training. The negative Poisson log-likelihood was chosen as the 404 objective function and $\ln (N \cdot L)$ of elements were used as offset (i.e., base_margin in XGBoost). 405 Other non-default parameters used in DriverPower were as follows: eta $=0.05$, max_depth=8, 406 subsample=0.6, max_delta_step=1.2, early_stop_rounds=5 and nrounds=5000. The fea407 ture importance for GBM is measured by the improvement in accuracy brought by a feature 408 across all trees. XGBoost returns feature importance that sums up to 1 for all features. We 409 also normalized the importance to a $[0,1]$ scale (i.e., importance relative to the most important 410 feature).

411 Evaluation of two BMR models. We evaluated both models with $1 \mathrm{Mb}$ autosome bins $412(n=2,521)$ and training genomic elements $(n=867,266)$ defined above. The $1 M b$ elements have 413 been used in many studies ${ }^{14,65,15}$. For both elements, we obtain the predicted mutation rate by 414 5-fold cross validation (CV). For $1 \mathrm{Mb}$ elements, we used 4-fold data for model training and 1-fold 415 data for model evaluation. For training elements, we use 1-fold data to train the model and the 416 rest to evaluate. As per previous work, we used $R^{2}$ score and Pearson's $r$ as evaluation metrics ${ }^{15}$. 417 Standard error of the mean (SEM) for $R^{2}$ and $r$ was calculated from 5-fold CV.

418 Calculation of element functional impact scores. Four different functional scores were used 419 in this analysis ${ }^{16,17,18,19}$. For CDS, CADD (SNVs and indels, v1.3), DANN (SNVs) and EIGEN 420 (SNVs) scores were used. CADD indel scores were generated with the CADD web interface for 421 all observed indels in the PCAWG dataset. For splice site, CADD and DANN scores were used. 422 For non-coding elements, the CADD, DANN and LINSIGHT (SNVs and indels) score were used. 423 Then the following steps were used to calculate the functional impact score per genomic element.

424 Firstly, raw scores were retrieved for all observed mutations in the dataset. Secondly, all raw 425 scores were converted to phred-like scores by $-10 \log _{10}\left(\mathrm{rank} / N_{m}\right)$, where $N_{m}$ is the number of 426 observed mutations having scores. Thirdly, for each genomic element, its functional score $S$ was 427 calculated as:

$$
S=\frac{1}{N} \sum_{i=1}^{N} \bar{s}_{i}
$$


where $N$ is the number of donors and $\bar{s}_{i}$ is the average functional impact score for the $i$ th donor.

Adjustment of the mutation count. To compensate for the unbalanced number of mutations among samples, instead of using the mutation count per element directly we used the geometric mean of mutation count and sample count. That is, we use the balanced count $y^{b}=\sqrt{y \cdot n_{d}}$ instead of $y$ directly for significance test, where $n_{d}$ is the number of mutated donors. Based on the motivation that not all mutations should be weighted the same, the balanced mutation count $y^{b}$ was then adjusted for nearly significant elements (raw q-value $<0.25$ ) by a functional weight $w$, that is $y^{f}=w \cdot y^{b}$, where $y^{f}$ is the functionally adjusted mutation count. For the element $j$, the functional weight $w_{j}$ was calculated based on its functional score $S_{j}$ and a threshold score $S_{T}$ :

$$
w_{j}=\frac{S_{j}}{S_{T}}=\frac{S_{j}}{-10 \log _{10} F}
$$

The threshold score $S_{T}$ is controlled by a single parameter $F$ between 0 and 1 , and can be interpreted as the fraction of functionally relevant variants among all observed variants. Parameter tuning of $F$ can be found at Supplementary Note 1.

Assessment of the element significance. For each element, we calculated $P\left(y^{b} \geq \hat{y}\right)$ as the raw p-value and $P\left(y^{f} \geq \hat{y}\right)$ as the function-adapted p-value. Since over-dispersion has been documented in burden based methods and can affect the driver discovery accuracy ${ }^{22}$, here we performed a regression-based overdispersion test for each tumour cohort using the training set $^{66}$. Based on the result of the overdispersion test, we calculated the raw and function-adpated p-values by following a binomial distribution or a negative binomial distribution:

$$
y^{b} \text { or } y^{f} \sim \begin{cases}\mathbf{N B}(\hat{y}, s \cdot \theta), & \text { if } p \leq 0.01 \\ \mathbf{B}\left(N \cdot L, \frac{\hat{y}}{N \cdot L}\right), & \text { otherwise }\end{cases}
$$

where $p$ and $\theta$ are the $\mathrm{p}$-value and dispersion parameter estimated from the overdispersion test, and $s$ is the scaling factor for $\theta$ used to accommodate the discrepancy between test and training set in terms of the dispersion level. We used $s=3$ for lymphomas and $s=1$ otherwise in this analysis.

Multiple testing correction. In all cases, q-values were generated by the Benjamini-Hochberg procedure $^{67}$. We chose $\mathrm{q}<0.1$ as the significant level and $\mathrm{q}<0.25$ as the nearly-significant level. For each element set, multiple testing correction was performed for each tumour cohort (cohort q-value) and across all tumour cohorts (global q-value). Cohort q-values were used in functional adjustment and global q-values were used to define the final driver list.

Generation of reference cancer drivers. Reference cancer drivers were used to benchmark the performance of DriverPower. Three reference sets were used: (1) the COSMIC Cancer Gene Census (v82, $\mathrm{n}=567$ ); (2) the PCAWG consensus driver candidates (PCAWG-consensus; $\mathrm{n}=157$ 
459 for coding and $n=26$ for non-coding); (3) the PCAWG raw integrated driver candidates (PCAWG460 raw; $n=193$ for coding and $n=79$ for non-coding). PCAWG-consensus (q-value post-filtering < 4610.1 ) is a set of highly confident non-coding drivers and subjected to multiple stringent filters 462 as described. PCAWG-raw (q-value pre-filtering $<0.1$ ) is a superset of PCAWG-consensus and 463 includes non-coding drivers that were not subjected to the filtering process. PCAWG-raw driver 464 candidates that are mutated in fewer than three samples were removed in this analysis. For 465 promoter and 5'UTR candidates in the PCAWG consensus drivers, we reversed the filtering for 466 overlapping elements (i.e., one element is selected over the overlapping element based on prior 467 knowledge). For example, we kept both the promoter and the overlapping 5'UTR of WDR74 in 468 this analysis; in the PCAWG consensus set, the WDR74 promoter is preferentially selected over 469 its 5'UTR.

470 Benchmarking of DriverPower. We compared coding and non-coding driver candidates 471 called by DriverPower to driver candidates called by six other published driver detection tools 472 (ActiveDriverWGS ${ }^{25}$, ExInAtor ${ }^{20}$, LARVA $^{22}$, MutSig $^{24}$, ncdDetect $^{21}$ and oncodriveFML ${ }^{23}$ ). Driver 473 calls for 26 single tumour cohorts (no Skin-Melanoma, Lymph-CLL and Lymph-BNHL) were re474 trieved from the PCAWG driver group. For each method, we removed driver candidates that are 475 mutated in fewer than three samples. We used precision (TP/ (TP+FP)), recall (TP/ (TP+FN)) 476 and F1-score (2*Precision*Recall/(Precision+Recall)) as performance metrics.

477 For CDS, we used the CGC gene set as the gold standard. For each method, true positive genes 478 were defined as genes presented in the gold standard set and the precision was then calculated as 479 the fraction of true positive genes among all called genes. For recall, since we can't accurately 480 know the expected set of driver genes that should be called for each tumour cohort in the dataset, 481 a lower-bound approximation was used instead. The lower-bound approximation was estimated 482 by taking the union of all true positive genes identified by each method and the recall was then 483 calculated as the fraction of true positive genes called among the lower-bound approximation.

484 For gene splice sites, the same gold standard gene set and benchmark method as CDS were used. 485 Due to data availability, the comparison was only performed for ncdDetect, oncodriveFML and 486 DriverPower.

487 For promoters, enhancers, 3'UTRs and 5'UTRs, because the number of non-coding driver candi488 dates is small, four element sets were benchmarked together. No data for ExInAtor is available 489 for this comparison. For each tumour cohort, true positive driver elements were defined as el490 ements called by at least three methods. The calculation of precision, recall and F1-score was 491 then identical as for the CDS and splice site.

492 Somatic copy number and structural variations analysis. We used SCNA (including GIS493 TIC2.0 results) and SV call sets released January $2017^{68}$. The copy number status (loss, neutral 494 or gain) of a region is classified based on the difference between the absolute copy number of the 495 region and the genome-wide ploidy of the donor. For gene-level structural variations, we calcu496 lated the number of breakpoints per gene (including CDS, splice sites, UTRs and promoters) per 497 donor. 
498 Differential expression analysis. We used the upper quartile normalized gene expression

499 (FPKM-UQ) released May 2016 ${ }^{69}$. When comparing the expression difference between two

500 groups of donors, we fitted the following quasi-Poisson family generalized linear model and then

501 employed the likelihood ratio test to obtain p-values for mutational status: FPKM-UQ MUT +

502 SCNA + [Tissue], where MUT is the mutational status ( 0 for unmutated donors and 1 for mu-

503 tated donors), SCNA is the somatic copy number status $(-1,0$ or 1 for copy number loss, neutral

504 or gain, respectively) and Tissue is the tumour tissue type. The tissue type was only used for

505 pan-cancer comparison for the adjustment of tumour types.

506 WES data analysis. We obtained two whole exome sequencing datasets through the Genomic 507 Data Common (GDC) ${ }^{70}$ : TCGA-PAAD (35,321 somatic mutations across 180 samples) and 508 TCGA-LIHA (56,208 somatic mutations across 364 samples). We chose public MuTect2 variants 509 from GDC. Variant coordinates were lifted from hg38 to hg19 with the UCSC liftOver tool. Only 510 CADD scores were used to detect drivers. For TCGA-PAAD, GBM models trained from Panc511 AdenoCA of the PCAWG data were used. For TCGA-LIHA, GBM models trained from Liver-HCC 512 were used.

513 Code availability. The source code for DriverPower (written mainly in Python 3) is available 514 at https://github.com/smshuai/DriverPower. It is distributed under GNU General Public 515 License 3.0, which allows for reuse and redistribution.

516 Data availability. The PCAWG data of simple somatic mutations, coding and non-coding 517 cancer driver calls, RNA expression values, copy number somatic mutations and structural somatic 518 mutations are available at the ICGC DCC (https://dcc.icgc.org/pcawg).

\section{Figures}

520 Figure 1 Summary of method and results. (a) DriverPower overview. (b,c) For the training 521 and test element sets, comparison of the predicted ( $\mathrm{Y}$-axis) and observed ( $\mathrm{X}$-axis) mutation rate 522 in the pan-cancer cohort. (d) The raw and function-adapted p-value quantile-quantile (QQ)523 plot for all test elements in the pan-cancer cohort. Function-adapted p-values are p-values with 524 the incorporation of functional impact scores. (e) Number and fraction of non-coding driver 525 candidates called by DriverPower contained within three reference driver sets (CGC, PCAWG526 consensus or PCAWG-raw). For each element type, the number of candidates is also shown 527 above the bar.

528 Figure 2 Benchmarking DriverPower driver discovery performance. (a) Comparison of 529 CDS results with or without functional adjustment for Panc-AdenoCA. Dashed lines in (a) repre530 sent the q-value $=0.1$ threshold. Function-adapted q-values are q-values with the incorporation 531 of functional impact scores. Only significant genes are labelled (color legend is the same as Fig. 532 1e). (b,c) Benchmark results for coding genes compared to six other driver discovery methods. 533 (d,e) Benchmark results for 3'UTR, 5'UTR, promoter and enhancer sets. (b) and (d) show the 534 precision and recall for each method according to results of 26 tumour cohorts (no melanoma 
535 and lymphoma). (c) shows the number and fraction of coding driver candidates called by each

536 method that are contained within reference gene sets. The colored columns in (c) correspond to 537 different reference driver sets (color legend is the same as Fig. 1e). (e) shows the number and 538 fraction of non-coding driver candidates called by each method that are also called by others. The 539 colored columns in (e) correspond to the number of methods that agree on a driver candidate. 540 (f) Differential expression analysis for the CDS and splice site of SGK1 in Lymph-BNHL. (g) 541 Differential expression analysis for the GPR126 enhancer in Bladder-TCC. MUT indicates sam542 ples with mutated element and WT indicates samples without mutated element. Copy number 543 corrected p-values from the likelihood ratio test and the $\log 2$ fold changes $(\log 2 \mathrm{FC})$ are shown in 544 blue. 


\section{Supplementary Information}

\section{Supplementary Notes}

\section{Supplementary Note 1 Parameter tuning}

548 As noted in the Method section, a single parameter $F$ (the functional score threshold) was 549 used to control the degree of functional adjustment in DriverPower. Functional scores used in 550 DriverPower were positively correlated with the functional impact of mutations within elements. 551 The parameter $F$ controls the threshold score $\left(S_{T}=-10 \log _{10} F\right)$ and must be within the interval $552(0,1]$. The parameter $F$ is negatively correlated with $S_{T}$. Genomic element with score $S>S_{T}$ 553 will be up-weighted; while element with score $S<S_{T}$ will be down-weighted. Since DriverPower 554 uses phred-scale scores, $F$ can be interpreted as the proportion of functionally relevant variants 555 among all observed variants. For instance, $F=0.01$ means $S_{T}=20$ so elements with functional 556 score $S>20$ (i.e., rank top $1 \%$ in phred-like scale) will receive a functional weight $>1$ and 557 gain additional significance from functional impact information. Using larger $F$ will result in 558 smaller $S_{T}$, and more elements will obtain additional significance, we hence expect to obtain 559 more driver candidates. Empirically speaking, larger $F$ will cause higher recall but lower precision 560 as illustrated in Supplementary Fig. 16.

561 The choice of $F$ is dependent on the score scheme in use and/or the element set and tumour 562 cohort in test. Here to avoid overfitting, we divided all 2583 donors into training donor set $563(\mathrm{~N}=1,117)$ and test donor set $(\mathrm{N}=1,136)$ to choose $F$ for four score schemes (CADD, DANN, 564 EIGEN and LINSIGHT) and three element types (CDS, splice site and other non-coding sites) 565 separately. For CDS and splice site, we used the COSMIC cancer gene census (CGC) as the gold 566 standard set for parameter tuning. For other non-coding sites, we used the PCAWG-raw as the

567

568 gold standard set for parameter tuning. Precisions were calculated as the fraction of hits in the gold standard set and pseudo-recalls were calculated as the number of significant candidates with functional adjustment in gold standard over the number of nearly-significant candidates without functional adjustment in gold standard. This calculation of pseudo-recall measured the relative performance of DriverPower to its personal best. We observed that optimal parameters for the training donor set also worked in a similar way for the test donor set. Parameters learnt here were used for driver discovery in this analysis.

\section{Supplementary Note 2 Difference from the PCAWG freeze}

The list of coding and non-coding drivers produced by this analysis (v1.0.0) differs slightly from the DriverPower results included in the PDFIG data analysis freeze due to the following reasons:

1. Hypermutated samples were not removed for the PCAWG freeze.

2. Only the binomial test was used in the PCAWG freeze.

3. The BMR model used in the PCAWG freeze was randomized lasso followed by GLM and in this analysis was gradient boosting machines. 


\section{Supplementary Tables}

594 Supplementary Table 1 Summary of tumour cohorts

595 Supplementary Table 2 Summary of genomic features

596 Supplementary Table 3 Coding and non-coding driver candidates called by DriverPower

597 Supplementary Table 4 DriverPower-exclusive driver candidates

\section{Supplementary Figures}

599 Supplementary Figure 1 Heterogeneity at cohort and donor levels. The violin plot shows 600 the number of somatic mutations (log-scale) per donor across all tumour types. Hypermutated 601 donors are included in the figure. Circles in the violin plot represent median values.

602 Supplementary Figure 2 Heterogeneity at the locus level. The average number of muta603 tions per cohort per $\mathrm{Mb}$ are shown for chromosome 22. Black lines show the mean mutation rate per $1 \mathrm{Mb}$ interval. Filled ribbons show the mutation rate within 1 standard deviation.

Supplementary Figure 3 Features correlation matrix. The heatmap shows the Spearman's rank correlation coefficient matrix between 1,373 features. Rows and columns are ordered by feature subgroups (40 subgroups in total). Barplot at right of the heatmap shows the Spearman's rho between features and mutation rates in training data for pan-cancer.

\section{Supplementary Figure 4 Expected versus GLM predicted mutation rate. Mutation rates} are the number of non-coding mutations within non-overlap $1 \mathrm{Mb}$ genome windows and normalized to per $\mathrm{Mb}$ per donor. Predictions are made from the randomized lasso and the generalized linear model. The $R^{2}$ (coefficient of determination) and $r$ (Pearson's correlation coefficients) are calculated with 5-fold cross validation. Note that only selected features are used in the GLM. 
614 Supplementary Figure 5 Expected versus GBM predicted mutation rate. Mutation rates

615 are the number of non-coding mutations within non-overlap $1 \mathrm{Mb}$ genome windows and normalized

616 to per $\mathrm{Mb}$ per donor. Predictions are made from the gradient boosting machines. The $R^{2}$

617 (coefficient of determination) and $r$ (Pearson's correlation coefficients) are calculated with 5-fold

618 cross validation. All features are used in the GBM.

619 Supplementary Figure 6 Model comparison. (a) Variance explained for all tumour cohorts 620 in $1 \mathrm{Mb}$ genome elements. (b) Variance explained for all tumour cohorts in the training element 621 set. Error bars show the standard error of the mean (SEM). SEM is calculated from 5-fold cross

622 validation. GBM is the gradient boosting machine. GLM is the generalized linear models.

623 Supplementary Figure 7 Feature importance rank. All features are divided into subgroups 624 (x-axis). Maximum importance of each group per tumour cohort (including pan-cancer) is repre625 sented as a point. Violin plot shows the distribution and median feature importance per subgroup 626 among tumour cohorts. (a) Feature importance measures by the randomized lasso. Red line shows 627 the 0.5 cutoff used in the generalized linear model. (b) Feature importance measures by the GBM 628 (log-scale).

629 Supplementary Figure 8 Top features associated with cell lines having similar origins. 630 Barplot shows the importance of 15 replication timing features for CNS-GBM, Liver-HCC and 631 Breast-AdenoCA. X-axis shows the cell line name where the repli-seq experiment is conducted 632 (ENCODE). Red line shows the 0.5 cutoff used in the feature selection. Feature weights from 633 the GBM are normalized to $[0,1]$.

634 Supplementary Figure 9 Elemental functional impact scores. (a) Functional impact scores 635 across all sets of elements for four different score schemes (CADD, DANN, EIGEN and LIN$636 \mathrm{SIGHT}$ ). Random elements are the training elements used in the model. (b) Spearman's corre637 lation matrix for each set of elements (pairwise complete).

638 Supplementary Figure 10 P-value quantile-quantile plots. The p-value QQ-plots are shown 639 for all test element types across three simulated datasets generated by the PDFIG and the observed 640 PCAWG dataset. For the observed dataset, both raw p-values (blue) and function-adapted p641 values (red) are shown. For the Broad, DKFZ and Sanger simulations, only raw p-values are 642 shown and elements with q-value $<0.1$ are labelled. Each QQ-plot contains p-values from all 643 tumour cohorts as the FDR control is performed in this way. Elements without mutations are 644 removed in the plot. For better visualization, - $\log 10$ observed p-values are capped at 16.

645 Supplementary Figure 11 Functional adjustment improves CDS driver discovery. Black 646 horizontal and vertical dashed lines show the q-value $=0.1$ cutoffs. Only significant genes are 647 labelled with colors corresponding to reference gene sets. Function-adapted q-values are q-values 648 with the usage of functional impact scores. Genes in the top-right quadrant are significant in both 649 raw and functional adjusted results, in the bottom-left quadrant are significant in neither. Genes 650 in the top-left are significant only after functional adjustment and genes in the bottom-right are 651 removed after functional adjustment.

652 Supplementary Figure 12 Comparison of DriverPower and MutSig for CDS results. The 
653 Venn diagram shows the relationship of true positive CDS results for DriverPower and Mut-

654 Sig. Significant genes present within the COSMIC CGC are considered to be true positive calls.

655 DriverPower identifies 21 and MutSig identified 23 true positive genes exclusively.

656 Supplementary Figure 13 Benchmark result of splice sites. The left panel shows the 657 precision and recall for each method according to the results on 26 tumour cohorts. The right 658 panel shows a heatmap of significant candidates identified by each method. Significant candidates 659 in CGC are filled with blue and not in CGC are filled with black.

660 Supplementary Figure 14 DriverPower-exclusive driver candidates overview. (a,b) CDS 661 of EEF1A2 in Eso-AdenoCA. (a) is a mutation lollipop plot of EEF1A2 in Eso-AdenoCA. (b) 662 shows the somatic copy number status for EEF1A2 in Eso-AdenoCA or non-Eso-AdenoCA (other) 663 samples; the p-value is from the Fisher's exact test. (c) A lollipop plot for CDS of MEF2B in 664 Lymph-BNHL. (d) A violin plot for differential expression between Lymph-BNHL samples with 665 mutated (MUT) MEF2B and non-mutated (WT) MEF2B. (e) Lollipop plots for CDS and splice 666 site of SGK1 in Lymph-BNHL. (f) Lollipop plots for GPR126 enhancer in Breast-AdenoCA and 667 Bladder-TCC. Lollipop plots show the distribution and classes of mutations (legends on top). 668 In the lollipop plot, element is shown as a rectangular box and blocks of a disjoint element are 669 separated by vertical dashed lines. Text within the box shows the name, type and length (bp) of 670 elements. Arrow below the element box indicates the direction of element.

671 Supplementary Figure 15 WES driver discovery result. P-value Q-Q plots for two TCGA 672 whole-exome sequencing datasets. Only significant genes are labelled $(q<0.1)$. Blue points and 673 labels indicate candidates within reference driver sets; red points and labels indicate likely false 674 positive hits.

675 Supplementary Figure 16 Parameterization of DriverPower. Each panel shows a parameter $676(F)$ search for one of the score schemes (CADD, DANN, EIGEN and LINSIGHT) and one of 677 the element sets (CDS, splice site and other non-coding elements). The best $F$ is indicated 678 below each panel title. For each search path, the left end point is the smallest $F$ searched and 679 the right end point is the largest $F$ searched; points correspond to $F$ searched and triangles 680 correspond to the best $F$. Search paths are colored for training (blue) and test (red) donor sets 681 (see Supplementary Note 1 for more details).

682 Supplementary Figure 17 Comparison of PCAWG freeze and v1.0.0. (a,b) Comparison 683 of coding driver candidates for non-melanoma/lymphoma tumours. (a) is the venn plot showing 684 the relationship between CDS results from PCAWG freeze and CDS results from this analysis 685 (v1.0.0). (b) shows the number and fraction of candidates called for two versions. Columns 686 in (b) are colored by reference gene sets. (c,d) Comparison of non-coding driver candidates for 687 non-melanoma/lymphoma tumours. (c) is the venn plot showing the relationship of non-coding 688 results (3'UTR, 5'UTR, promoter, enhancer, IncRNA and IncRNA promoter) between PCAWG 689 freeze and v1.0.0. (d) shows all non-coding candidates that are unique to PCAWG freeze $(n=7)$ 690 or v1.0.0 $(n=4)$. See Supplementary Note 2 for more details. 


\section{References}

6921 Stratton MR, Campbell PJ, Futreal PA. The cancer genome. Nature 2009; 458: 719-724.

6932 Vogelstein B, Papadopoulos N, Velculescu VE, Zhou S, Diaz LA, Kinzler KW. Cancer Genome 694 Landscapes. Science 2013; 339: 1546-1558.

6953 Khurana E, Fu Y, Chakravarty D, Demichelis F, Rubin MA, Gerstein M. Role of non-coding 696 sequence variants in cancer. Nature Reviews Genetics 2016; 17: 93-108.

6974 Garraway LA, Lander ES. Lessons from the Cancer Genome. Cell 2013; 153: 17-37.

6985 Martincorena I, Campbell PJ. Somatic mutation in cancer and normal cells. Science 2015; 699 349: 1483-1489.

7006 Lawrence MS, Stojanov P, Polak P, Kryukov GV, Cibulskis K, Sivachenko A et al.. Mutational 701 heterogeneity in cancer and the search for new cancer-associated genes. Nature 2013; 499: 702 214-218.

7037 Mansour MR, Abraham BJ, Anders L, Berezovskaya A, Gutierrez A, Durbin AD et al.. Onco704 gene regulation. An oncogenic super-enhancer formed through somatic mutation of a noncoding 705 intergenic element. Science 2014; 346: 1373-7.

7068 Huang FW, Hodis E, Xu MJ, Kryukov GV, Chin L, Garraway LA. Highly recurrent TERT 707 promoter mutations in human melanoma. Science 2013; 339: 957-9.

7089 Pan-cancer analysis of whole genomes. bioRxiv 2017. doi:10.1101/162784.

70910 Kundaje, Meuleman, Ernst, Bilenky, Yen, Heravi-Moussavi et al.. Integrative analysis of 111 710 reference human epigenomes. Nature 2015; 518: 317-330.

71111 Dunham, Kundaje, Aldred, Collins, Davis, Doyle et al.. An integrated encyclopedia of DNA 712 elements in the human genome. Nature 2012; 489: 57-74.

71312 Rosenbloom KR, Armstrong J, Barber GP, Casper J, Clawson H, Diekhans M et al.. The 714 UCSC Genome Browser database: 2015 update. Nucleic Acids Research 2015; 43: D681.

71513 Friedman JH. Greedy function approximation: a gradient boosting machine. Annals of statis716 tics 2001; : 1189-1232.

71714 Schuster-Böckler B, Lehner B. Chromatin organization is a major influence on regional muta718 tion rates in human cancer cells. Nature 2012; 488: 504-507.

71915 Polak, Karlić, Koren, Thurman, Sandstrom, Lawrence et al.. Cell-of-origin chromatin organi720 zation shapes the mutational landscape of cancer. Nature 2015; 518: 360-364.

72116 Kircher M, Witten DM, Jain P, O'Roak BJ, Cooper GM, Shendure J. A general framework 722 for estimating the relative pathogenicity of human genetic variants. Nature Genetics 2014; 46: $723 \quad 310-315$. 
72417 Quang D, Chen Y, Xie X. DANN: a deep learning approach for annotating the pathogenicity

725 of genetic variants. Bioinformatics 2015; 31: 761-763.

72618 Ionita-Laza I, McCallum K, Xu B, Buxbaum JD. A spectral approach integrating functional 727 genomic annotations for coding and noncoding variants. Nature Genetics 2016; 48: 214-220.

72819 Huang Y-F, Gulko B, Siepel A. Fast, scalable prediction of deleterious noncoding variants from 729 functional and population genomic data. Nature Genetics 2017; 49: 618-624.

73020 Lanzós A, Carlevaro-Fita J, Mularoni L, Reverter F, Palumbo E, Guigó R et al.. Discovery 731 of Cancer Driver Long Noncoding RNAs across 1112 Tumour Genomes: New Candidates and 732 Distinguishing Features. Scientific Reports 2017; 7: 41544.

73321 Juul M, Bertl J, Guo Q, Nielsen MM, Świtnicki M, Hornshøj H et al.. Non-coding cancer 734 driver candidates identified with a sample- and position-specific model of the somatic mutation 735 rate. elife 2017 ; 6: e21778.

73622 Lochovsky L, Zhang J, Fu Y, Khurana E, Gerstein M. LARVA: an integrative framework for 737 large-scale analysis of recurrent variants in noncoding annotations. Nucleic acids research 2015; 738 : gkv803.

73923 Mularoni L, Sabarinathan R, Deu-Pons J, Gonzalez-Perez A, López-Bigas N. OncodriveFML: 740 a general framework to identify coding and non-coding regions with cancer driver mutations. 741 Genome biology 2016; 17: 128.

74224 Rheinbay E, Parasuraman P, Grimsby J, Tiao G, Engreitz JM, Kim J et al.. Recurrent and 743 functional regulatory mutations in breast cancer. Nature 2017; 547: 55-60.

74425 Reimand J. Abstract 385: Network-driven discovery of cancer drivers and pathways using 745 2,500 whole cancer genomes. Cancer research 2017; 77: 385.

74626 Futreal PA, Coin L, Marshall M, Down T, Hubbard T, Wooster R et al.. A census of human 747 cancer genes. Nature Reviews Cancer 2004; 4: 177-183.

74827 Forbes SA, Beare D, Gunasekaran P, Leung K, Bindal N, Boutselakis H et al.. COSMIC: 749 exploring the world's knowledge of somatic mutations in human cancer. Nucleic acids research 750 2015; 43: D811.

75128 Roberts SA, Gordenin DA. Hypermutation in human cancer genomes: footprints and mecha752 nisms. Nature Reviews Cancer 2014; 14: 786-800.

75329 Sabarinathan R, Mularoni L, Deu-Pons J, Gonzalez-Perez A, López-Bigas N. Nucleotide exci754 sion repair is impaired by binding of transcription factors to DNA. Nature 2016; 532: 264-267.

75530 Perera D, Poulos RC, Shah A, Beck D, Pimanda JE, Wong JWH. Differential DNA repair 756 underlies mutation hotspots at active promoters in cancer genomes. Nature 2016; 532: 259-263.

75731 Porter LA, Collins C, Gray JW, Lee JM, Murthy S, Cukier IH et al.. Protein elongation factor 758 EEF1A2 is a putative oncogene in ovarian cancer. Nature Genetics 2002; 31: 301-305. 
32 Tomlinson VAL, Newbery HJ, Wray NR, Jackson J, Larionov A, Miller WR et al.. Translation elongation factor eEF1A2 is a potential oncoprotein that is overexpressed in two-thirds of breast tumours. BMC cancer 2005; 5: 113.

33 L. Tomlinson V a., Newbery HJ, Bergmann JH, Boyd J, Scott D, Wray NR et al.. Expression of eEF1A2 is associated with clear cell histology in ovarian carcinomas: overexpression of the gene is not dependent on modifications at the locus. British Journal of Cancer 2007; 96: 1613-1620.

34 Zhang J, Grubor V, Love CL, Banerjee A, Richards KL, Mieczkowski PA et al.. Genetic heterogeneity of diffuse large B-cell lymphoma. Proceedings of the National Academy of Sciences 2013; 110: 1398-1403.

35 Lohr JG, Stojanov P, Lawrence MS, Auclair D, Chapuy B, Sougnez C et al.. Discovery and prioritization of somatic mutations in diffuse large B-cell lymphoma (DLBCL) by whole-exome sequencing. Proceedings of the National Academy of Sciences of the United States of America 2012; 109: 3879-3884.

36 Reddy A, Zhang J, Davis NS, Moffitt AB, Love CL, Waldrop A et al.. Genetic and Functional Drivers of Diffuse Large B Cell Lymphoma. Cell 2017; 171: 481-494.e15.

37 Pon JR, Wong J, Saberi S, Alder O, Moksa M, Cheng S-WG et al.. MEF2B mutations in non-Hodgkin lymphoma dysregulate cell migration by decreasing MEF2B target gene activation. Nature Communications 2015; 6: 7953.

38 Hartmann S, Schuhmacher B, Rausch T, Fuller L, Döring C, Weniger M et al.. Highly recurrent mutations of SGK1, DUSP2 and JUNB in nodular lymphocyte predominant Hodgkin lymphoma. Leukemia 2016; 30: 844-853.

39 Veer LJ van 't, Dai H, Vijver MJ van de, He YD, Hart AAM, Mao M et al.. Gene expression profiling predicts clinical outcome of breast cancer. Nature 2002; 415: 530-536.

40 Tian S, Roepman P, Van't VLJ, Bernards R, de SF, Glas AM. Biological functions of the genes in the mammaprint breast cancer profile reflect the hallmarks of cancer.. Biomark Insights 2010; 5: 129-38.

41 Cui H, Wang Y, Huang H, Yu W, Bai M, Zhang L et al.. GPR126 protein regulates developmental and pathological angiogenesis through modulation of VEGFR2 receptor signaling. Journal of Biological Chemistry 2014; 289: 34871-34885.

42 Wu G, Broniscer A, McEachron TA, Lu C, Paugh BS, Becksfort J et al.. Somatic histone H3 alterations in pediatric diffuse intrinsic pontine gliomas and non-brainstem glioblastomas. Nature Genetics 2012; 44: 251-253.

43 Schwartzentruber J, Korshunov A, Liu X-Y, Jones DTW, Pfaff E, Jacob K et al.. Driver mutations in histone H3. 3 and chromatin remodelling genes in paediatric glioblastoma. Nature 2012; 482: 226-231.

44 Fang D, Gan H, Lee J-H, Han J, Wang Z, Riester SM et al.. The histone H3. 3K36M mutation 
795 reprograms the epigenome of chondroblastomas. Science 2016; 352: 1344-1348.

79645 Su X, Lucas DM, Zhang L, Xu H, Zabrouskov V, Davis ME et al.. Validation of an LCMS 797 based approach for profiling histones in chronic lymphocytic leukemia. Proteomics 2009; 9: 798 1197-1206.

79946 Singh R, Mortazavi A, Telu KH, Nagarajan P, Lucas DM, Thomas-Ahner JM et al.. Increasing 800 the complexity of chromatin: functionally distinct roles for replication-dependent histone $\mathrm{H} 2 \mathrm{~A}$ 801 isoforms in cell proliferation and carcinogenesis. Nucleic Acids Research 2013; 41: 9284-9295.

80247 Dou Y, Milne TA, Tackett AJ, Smith ER, Fukuda A, Wysocka J et al.. Physical Association 803 and Coordinate Function of the H3 K4 Methyltransferase MLL1 and the H4 K16 Acetyltransferase 804 MOF. Cell 2005; 121: 873-885.

80548 Sykes SM, Mellert HS, Holbert MA, Li K, Marmorstein R, Lane WS et al.. Acetylation of the 806 p53 DNA-Binding Domain Regulates Apoptosis Induction. Molecular Cell 2006; 24: 841-851.

80749 Gupta A, Sharma GG, Young CSH, Agarwal M, Smith ER, Paull TT et al.. Involvement of 808 Human MOF in ATM Function. Molecular and Cellular Biology 2005; 25: 5292-5305.

80950 Rea S, Xouri G, Akhtar A. Males absent on the first (MOF): from flies to humans. Oncogene 810 2007; 26: 5385-5394.

81151 ZHU LIN, YANG JIAXING, ZHAO LINHONG, YU XUE, WANG LINGYAO, WANG FEl et al.. 812 Expression of hMOF but not HDAC4 is responsible for the global histone H4K16 acetylation in 813 gastric carcinoma. International Journal of Oncology 2015; 46: 2535-2545.

81452 Luo H, Shenoy AK, Li X, Jin Y, Jin L, Cai Q et al.. MOF Acetylates the Histone Demethylase 815 LSD1 to Suppress Epithelial-to-Mesenchymal Transition. Cell Reports 2016; 15: 2665-2678.

$81653 \mathrm{Fu} \mathrm{Y,} \mathrm{Huang} \mathrm{B,} \mathrm{Shi} \mathrm{Z,} \mathrm{Han} \mathrm{J,} \mathrm{Wang} \mathrm{Y,} \mathrm{Huangfu} \mathrm{J} \mathrm{et} \mathrm{al..} \mathrm{SRSF1} \mathrm{and} \mathrm{SRSF9} \mathrm{RNA} \mathrm{bind-}$ 817 ing proteins promote Wnt signallingmediated tumorigenesis by enhancing catenin biosynthesis. 818 EMBO molecular medicine 2013; 5: 737-750.

81954 Araya CL, Cenik C, Reuter JA, Kiss G, Pande VS, Snyder MP et al.. Identification of signif820 icantly mutated regions across cancer types highlights a rich landscape of functional molecular 821 alterations. Nature Genetics 2016; 48: 117-125.

82255 Castro-Giner F, Ratcliffe P, Tomlinson I. The mini-driver model of polygenic cancer evolution. 823 Nature Reviews Cancer 2015; 15: 680-685.

82456 McFarland CD, Korolev KS, Kryukov GV, Sunyaev SR, Mirny LA. Impact of deleterious 825 passenger mutations on cancer progression. Proceedings of the National Academy of Sciences 826 2013; 110: 2910-2915.

82757 Kumar S, Gerstein M. Cancer genomics: Less is more in the hunt for driver mutations. Nature 828 2017; 547: 40-41. 
82958 Harrow, Frankish, Gonzalez, Tapanari, Diekhans, Kokocinski et al.. GENCODE: The reference

830 human genome annotation for The ENCODE Project. Genome Research 2012; 22: 1760-1774.

83159 Kent WJ, Zweig AS, Barber G, Hinrichs AS, Karolchik D. BigWig and BigBed: enabling 832 browsing of large distributed datasets. Bioinformatics 2010; 26: 2204-2207.

83360 Quinlan AR, Hall IM. BEDTools: a flexible suite of utilities for comparing genomic features.

834 Bioinformatics 2010; 26: 841-842.

83561 Pedregosa F, Varoquaux G, Gramfort A, Michel V, Thirion B, Grisel O et al.. Scikit-learn:

836 Machine Learning in Python. Journal of Machine Learning Research 2011; 12: 2825-2830.

83762 Rosenbloom KR, Sloan CA, Malladi VS, Dreszer TR, Learned K, Kirkup VM et al.. ENCODE

838 data in the UCSC Genome Browser: year 5 update. Nucleic acids research 2012; 41: D63.

83963 Meinshausen N, Bühlmann P. Stability selection. Journal of the Royal Statistical Society Series

840 B (Statistical Methodology) 2010; 72: 417-473.

84164 Chen T, Guestrin C. XGBoost: A Scalable Tree Boosting System. In: Proceedings of the 842 22nd acm sigkdd international conference on knowledge discovery and data mining. ACM: New 843 York, NY, USA, 2016, pp 785-794.

84465 Hellmann I, Prüfer K, Ji H, Zody MC, Pääbo S, Ptak SE. Why do human diversity levels vary 845 at a megabase scale?. Genome Research 2005; 15: 1222-1231.

84666 Cameron AC, Trivedi PK. Regression-based tests for overdispersion in the Poisson model. 847 Journal of Econometrics 1990; 46: 347-364.

84867 Benjamini Y, Hochberg Y. Controlling the false discovery rate: a practical and powerful 849 approach to multiple testing. Journal of the Royal Statistical SocietySeries B (Methodological) 850 1995; : 289-300.

$85168 \mathrm{Li}$ Y, Roberts N, Weischenfeldt J, Wala JA, Shapira O, Schumacher S et al.. Patterns of 852 structural variation in human cancer. bioRxiv 2017. doi:10.1101/181339.

85369 Pan-cancer study of heterogeneous RNA aberrations. bioRxiv 2017. doi:10.1101/183889.

85470 Grossman RL, Heath AP, Ferretti V, Varmus HE, Lowy DR, Kibbe WA et al.. Toward a shared 855 vision for cancer genomic data. New England Journal of Medicine 2016; 375: 1109-1112. 
a

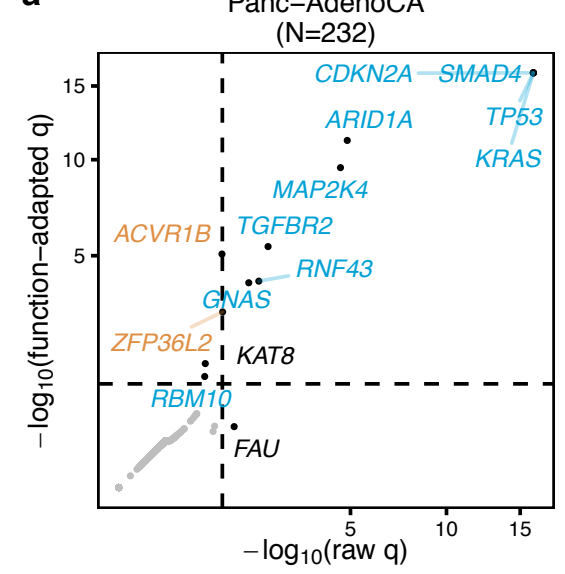

d

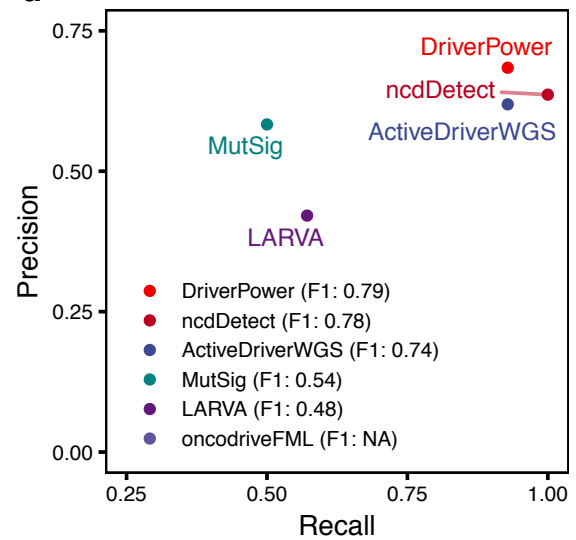

b Protein coding elements

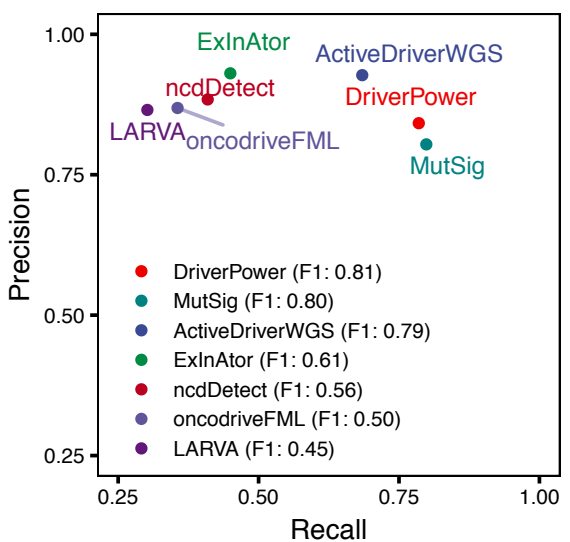

e

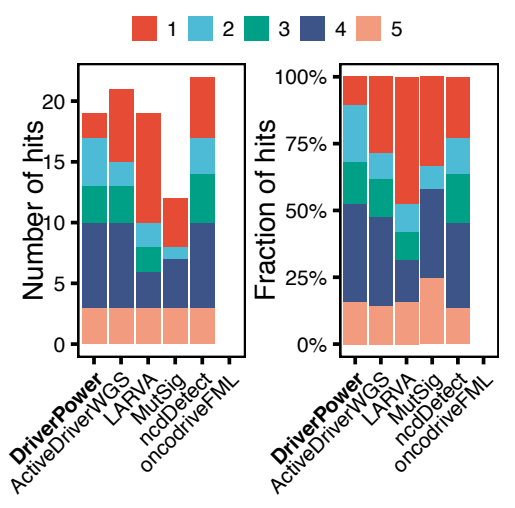

C
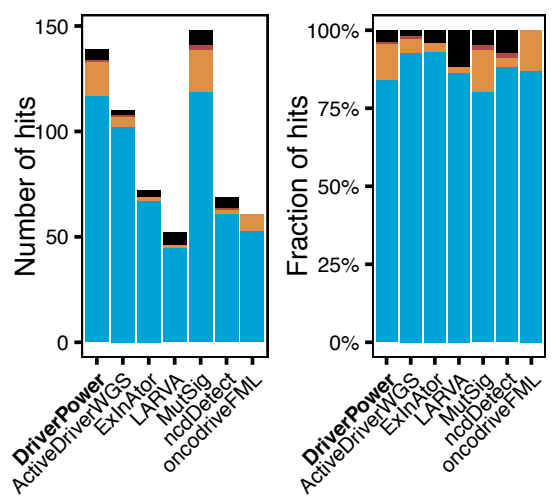

f

g

Lymph-BNHL SGK1

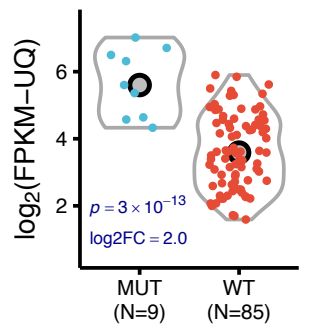

Bladder-TCC GPR126

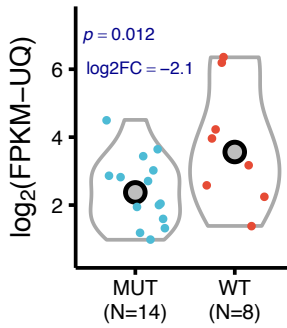

Figure 2 